\title{
Bosque seco de la parroquia Mangahurco, Zapotillo, Loja, estudio de su composición florística, estructura y endemismo
}

\author{
Zhofre Aguirre Mendoza \\ zhofre.aguirre@unl.edu.ec \\ Docente-Investigador, \\ Universidad Nacional de Loja, Ecuador \\ Carmen Aponte Córdova \\ c_ma_aponte@hotmail.es \\ Ingeniera Forestal, Libre ejercicio profesional, \\ Loja, Ecuador \\ Wilson Quizhpe Coronel \\ wr.quizhpec@uea.edu.ec \\ Docente-Investigador, \\ Universidad Estatal Amazónica, El Pangui, Ecuador
}

\section{RESUMEN}

Los bosques secos en Ecuador son ecosistemas con importante diversidad florística y faunística y son parte de la zona de endemismo Tumbesino. Este estudio se realizó en la parroquia Mangahurco, cantón Zapotillo, provincia de Loja, con el objetivo de determinar la composición florística y estructura del bosque seco. Se estableció una parcela permanente de $100 \times 100$ m, anidando 25 subparcelas de 20 × 20 m, en cada subparcela, se registraron todos los individuos $\geq 5 \mathrm{~cm}$ de $\mathrm{D}_{1,30 \mathrm{~m}}$, los arbustos, hierbas y epifitas. Se calculó el índice de Shannon, parámetros estructurales, área basal y volumen. Se inventariaron 1117 individuos/ha que pertenecen a 61 especies, 32 son árboles, 7 arbustos, 18 hierbas y 4 epífitas. Las familias más diversas son: Fabaceae, Asteraceae, Bignoniaceae, Malvaceae y Verbenaceae. El índice de Shanon es de 0,38 para árboles, 0,26 para arbustos y 0,42 para hierbas. Se reporta una especie endémica para Ecuador y nueve endémicas compartidas para la Región Tumbesina. Las especies con mayor IVI son: Handroanthus chrysanthus, Simira ecuadorensis y Citharexylum gentryi. Se diferencian tres estratos, en el estrato superior Cochlospermum vitifolium, Eriotheca ruizii, Ceiba trichistandra, Terminalia valverdae y Handroanthus chrysanthus, en el estrato codominante Simira ecuadorensis, Chloroleucon mangense, Celtis loxensis, Caesalpina glabrata y Geoffroea spinosa y en el estrato suprimido Celtis loxensis, 
Pisonia aculeata y Cynophylla sclerophylla. El bosque tiene un área basal de $16,99 \mathrm{~m}^{2} / \mathrm{ha}$ y volumen de $61,11 \mathrm{~m}^{3} / \mathrm{ha}$. La composición florística y las características estructurales indican que el bosque se encuentra en buen estado de conservación.

Palabra claves: composición florística; estructura; endemismo; región tumbesina; índice de valor de importancia. 


\title{
Dry forest of the Mangahurco parish, Zapotillo, Loja, study of its floristic composition, structure and endemism
}

\begin{abstract}
The dry forests in Ecuador are ecosystems with important flora and fauna diversity and are part of the Tumbesian endemic zone. This study was carried out in the Mangahurco parish, Zapotillo canton, Loja province, with the objective of determining the floristic composition and structure of the dry forest. A permanent plot of $100 \times 100 \mathrm{~m}$ was established, nesting 25 subplots of $20 \times 20 \mathrm{~m}$, in each subplot, all individuals $\geq 5 \mathrm{~cm}$ of $\mathrm{D}_{1.30 \mathrm{~m}}$, shrubs, herbs and epiphytes were recorded. The Shannon index, structural parameters, basal area and volume were calculated. 1117 individuals / ha belonging to 61 species were inventoried, 32 are trees, 7 shrubs, 18 herbs and 4 epiphytes. The most diverse families are: Fabaceae, Asteraceae, Bignoniaceae, Malvaceae and Verbenaceae. The Shanon index is 0,38 for trees, 0,26 for shrubs, and 0,42 for herbs. One endemic species is reported for Ecuador and nine shared endemics for the Tumbesian Region. The species with the highest IVI are: Handroanthus chrysanthus, Simira ecuadorensis and Citharexylum gentryi. Three strata are differentiated, in the upper stratum Cochlospermum vitifolium, Eriotheca ruizii, Ceiba trichistandra, Terminalia valverdae and Handroanthus chrysanthus, in the codominant stratum Simira ecuadorensis, Chloroleucon mangense, Celtis loxensis, Caesalpina glabrata y Geoffroea spinosa, and the stratum suppressed Celtis loxensis, Pisonia aculeata y Cynophylla sclerophylla. The forest has a basal area of $16,99 \mathrm{~m}^{2} /$ ha and a volume of $61,11 \mathrm{~m}^{3} / \mathrm{ha}$. The floristic composition and structural characteristics indicate that the forest is in a good state of conservation
\end{abstract}

Keywords: floristic composition; structure; endemism; tumbes region; importance value index.

Artículo recibido: 10 Agosto. 2021 Aceptado para publicación: 07. Setiembre. 2021 Correspondencia: zhofre.aguirre@unl.edu.ec Conflictos de Interés: Ninguna que declarar 


\section{INTRODUCCIÓN}

Los bosques secos son formaciones vegetales deciduas, que presentan características muy particulares, donde más del $75 \%$ de las especies vegetales pierden estacionalmente sus hojas (Aguirre et al., 2006; Linares, 2004; Aguirre, 2013), la precipitación anual fluctúa entre 350-800 mm con una temporada seca de al menos cinco a seis meses, siendo una vegetación muy frágil, que se desarrolla en condiciones climáticas extremas (Pennington et al., 2000). Klitgaard et al. (1999) y Lamprecht (1990) indica que a nivel mundial existen aproximadamente 530 millones de hectáreas de bosques secos, en América Latina están distribuidos desde el norte de México hasta el sur de Brasil y constituyen el 66,7 \% de la superficie total (Linares, 2004).

En Ecuador los bosques secos costeros forman parte de la región Tumbesina del pacifico ecuatorial, compartido entre Ecuador y Perú, abarcan $135000 \mathrm{~km}^{2}$, ubicados al suroeste Ecuatoriano y noroeste Peruano desde 0 hasta 1000 m s.n.m (Aguirre et al., 2006; Aguirre, 2013).

Los bosques secos de Ecuador se encuentran ubicados en dos áreas: a) sobre la costa pacífica centro: Esmeraldas, Manabí, Santa Elena, Guayas; y, b) en la costa sur y estribaciones occidentales de los Andes: El Oro y Loja, ecosistema único en el mundo (Aguirre, 2013).

Los bosques secos del Ecuador y en particular la provincia de Loja, tradicionalmente ha sido sobreexplotado y degradado por la extracción de madera, ampliación de la frontera agrícola, incendios forestales, sobrepastoreo de ganado caprino y bovino, la cacería y la aplicación inadecuada de métodos de cosecha de miel (Paladines, 2003) todas estas actividades antrópicas han ocasionado cambios en la composición florística, estructura y dinámica del bosque seco.

En el área occidental de la provincia de Loja existen zonas boscosas poco intervenidas, como es el caso de la zona de Mangahurco, Bolaspamba y Cazaderos. Por esta razón se ha realizado la presente investigación en una parcela permanente, con el propósito de generar información sobre composición florística, estructura, que permitan plantear estrategias de conservación de estos bosques.

Este documento contiene información científica - técnica sobre la composición florística, parámetros estructurales, perfiles estructurales, parámetros dasométricos y volumétricos, índice de diversidad de Shannon y endemismo del bosque seco del sector El Tabanco. 


\section{MATERIALES Y MÉTODOS}

\section{Área de estudio}

La investigación se realizó en el sector El Tabanco, parroquia Mangahurco, cantón Zapotillo en la provincia de Loja, a una altitud de $627 \mathrm{msnm}$ (Figura 1); ubicada en las coordenadas UTM: Vértice 1: longitud: 564595 E, Latitud 9545378 N; vértice 2: longitud 564582, latitud E9 545282N; vértice 3: longitud $582685 \mathrm{E}$, latitud $95545306 \mathrm{~N}$ y punto 4: longitud 564636 E y latitud 9545385 N. El sector donde se ejecutó la investigación abarca una área aproximada de 100 ha.

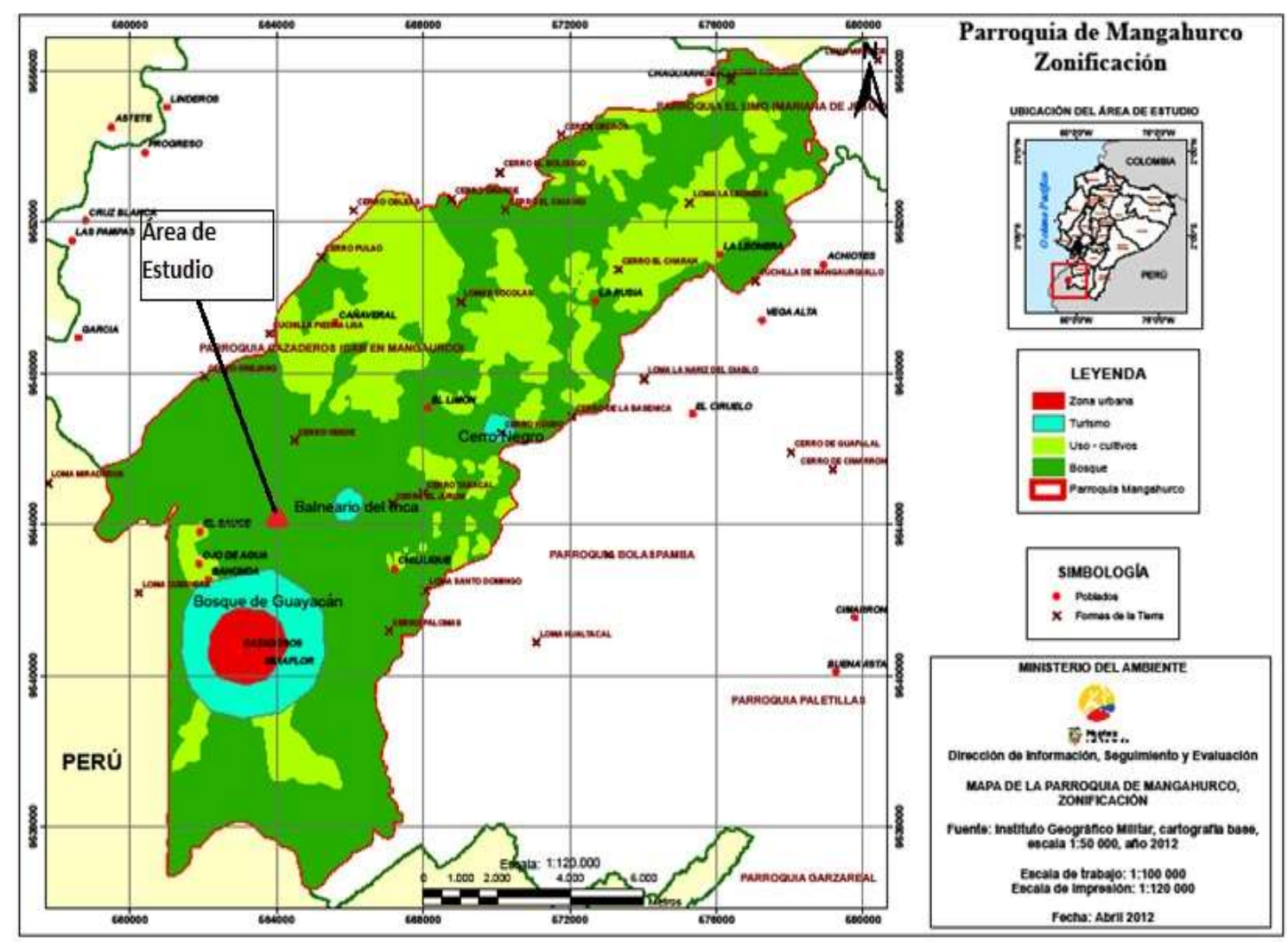

Figura 1. Mapa de ubicación de la zona de estudio del bosque seco del sector "El Tabanco”, parroquia Mangahurco, Zapotillo, Ecuador.

\section{Aspectos ecológicos}

Ésta zona de estudio posee un clima seco con una temperatura promedio de $24{ }^{\circ} \mathrm{C}$ y precipitaciones de 400-600 mm/año. Según el Herbario LOJA et al. (2001) la zona pertenece a la zona de vida bosque espinoso tropical (be-T) y según el MAE (2013) es un bosque deciduo de tierras del Jama-Zapotillo. El área es reconocida por la abundancia 
natural y el florecimiento de los guayacanes (Handroanthus chrysanthus) que se presenta en los meses de enero y/o febrero.

\section{Composición y diversidad florística}

Se instaló una parcela permanente de 1 ha $(100$ x 100 m) delimitada con brújula y piola, ubicando mojones de cemento y tubos PVC en las esquinas. Se subdividió en 25 subparcelas de $400 \mathrm{~m}^{2}$ (20 x $20 \mathrm{~m}$ ), dentro de éstas se midieron todos los individuos mayores a $5 \mathrm{~cm}$ de $\mathrm{D}_{1,30 \mathrm{~m}}$. Los individuos fueron etiquetados con placas metálicas y marcados con un código alfanumérico a la altura de 1,45 m. Los arbustos y hierbas se registraron en 9 subparcelas de $25 \mathrm{~m}^{2}(5 \times 5 \mathrm{~m})$ y $1 \mathrm{~m}^{2}(1 \mathrm{x} 1 \mathrm{~m})$ respectivamente. También, se inventariaron las epífitas vasculares, considerando como unidades de muestreo 1 árbol por cada parcela, siguiendo recomendaciones de Phillips et al. (2016).

Se colectaron muestras botánicas fértiles de las especies para su identificación en el Herbario "Reinaldo Espinosa" de la Universidad Nacional de Loja

\section{Cálculo de parámetros estructurales}

Se calculó los parámetros estructurales de la vegetación, utilizando las fórmulas recomendadas por Aguirre (2019) y Cerón (2003).

$$
\text { Densidad absoluta (D) \# ind } / \mathrm{m}^{2}=\frac{\mathrm{N}^{\circ} \text { total de individuos por especie }}{\text { Total del área muestreada }}
$$

Densidad relativa (DR) $\%=\frac{\mathrm{N}^{\circ} \text { de individuos por especie }}{\mathrm{N}^{\circ} \text { total de individuos }} \times 100$

Dominancia relativa $(\mathrm{DmR}) \%=\frac{\text { Área basal de la especie }}{\text { Área basal de todas las especies }} \times 100$

$$
\text { Frecuencia }(\mathrm{Fr})=\frac{\text { Número de cuadrantes en que está la especie }}{\text { Número total de cuadrantes evaluados }} \times 100
$$

Indice valor importanci a (IVI)\% $=(D R+D m R+F r) / 3$ 
Para determinar la diversidad alfa se utilizó el índice de Shannon (1) usando la fórmula:

$$
H=\sum_{i=1}^{S}(P i)\left(\log _{2} P i\right)
$$

\section{Cálculo de parámetros dasométricos}

Los individuos mayores a $5 \mathrm{~cm}$ de DAP, se agruparon en 10 clases diamétricas. Para calcular el factor de forma se seleccionó al azar tres árboles por clase diamétrica, que fueron medidos en pie en secciones de 1,5 m. El volumen de los árboles se calculó con la fórmula de Smalian (Aguirre, 2019).

$$
\begin{aligned}
& V a=\frac{G o+G 1}{2} \times L_{1}+\frac{G 1+G 2}{2} \times L_{2}+\frac{G 2+\ldots G n}{2} \times L_{n} \\
& V c=G \times H T \\
& f=\frac{V a}{V c} \\
& V=G \times H \times f
\end{aligned}
$$

\section{RESULTADOS}

\section{Composición florística}

Se registran 61 especies, dentro de 60 géneros y 34 familias, de los cuales 32 son árboreos incluidos en 32 géneros y 18 familias; 7 especies arbustivas que pertenecen a 6 géneros y 6 familias; en el estrato herbáceo 18 especies de 18 géneros y 8 familias y cinco especies de epífitas que pertenecen a 4 géneros y 2 familias (Anexo 1). Las familias del bosque con mayor diversidad de especies son: Fabaceae con 15 especies, Asteraceae con seis, Malvaceae con cinco y Rubiaceae con 3 especies.

\section{Parámetros estructurales del estrato arbóreo}

Se registraron 1117 individuos/ha mayores a $5 \mathrm{~cm}$ de DAP que pertenecen a 32 especies, 32 géneros y 18 familias. Los parámetros de las diez especies sobresalientes de este bosque se presentan en la Tabla 1 . 
Tabla 1. Parámetros estructurales de las diez especies sobresalientes de la parcela permanente del bosque seco del sector El Tabanco, Mangahurco.

\begin{tabular}{|l|l|c|c|c|c|c|}
\hline \multicolumn{1}{|c|}{ Familia } & \multicolumn{1}{|c|}{ Nombre Científico } & $\begin{array}{c}\text { D } \\
\text { Ind./ha }\end{array}$ & $\begin{array}{c}\text { Dr } \\
(\boldsymbol{\%})\end{array}$ & $\begin{array}{c}\text { DmR } \\
(\boldsymbol{\%})\end{array}$ & $\begin{array}{c}\text { Fr } \\
(\boldsymbol{\%})\end{array}$ & $\begin{array}{c}\text { IVI } \\
(\boldsymbol{\%})\end{array}$ \\
\hline Bignoniaceae & Handroanthus chrysanthus (Jacq.) S. O. Grose & 173 & 15,49 & 23,26 & 7,96 & 15,57 \\
\hline Rubiaceae & Simira ecuadorensis (Standl.) Steger & 246 & 22,02 & 0,13 & 7,64 & 9,93 \\
\hline Verbenaceae & Citharexylum gentryi Moldenke & 121 & 10,83 & 3,01 & 7,32 & 7,06 \\
\hline Malvaceae & Eriotheca ruizii (K. Schum.) A. Robyns & 18 & 1,61 & 11,58 & 3,82 & 5,67 \\
\hline Fabaceae & Chloroleucon mangense (Jacq.) Britton \& Rose & 81 & 7,25 & 3,99 & 5,73 & 5,66 \\
\hline Ulmaceae & Celtis loxensis Cc. Berg & 87 & 7,79 & 3,04 & 5,1 & 5,31 \\
\hline Bixaceae & Cochlospermum vitifolium (Willd.) Spreng. & 26 & 2,33 & 9,33 & 3,82 & 5,16 \\
\hline Malvaceae & Ceiba trichistandra (A. Gray) Bakh. & 5 & 0,45 & 12,91 & 1,59 & 4,98 \\
\hline Fabaceae & Machaerium millei Standl. & 49 & 4,39 & 3,84 & 6,05 & 4,76 \\
\hline Combretaceae & Terminalia valverdae A.H. Gentry & 47 & 4,21 & 4,86 & 4,78 & 4,62 \\
\hline
\end{tabular}

$D=$ Densidad $;$ DR= Densidad Relativa $; G=$ Área Basal; DmR=Dominancia Relativa $; F$ $a b=$ Frecuencia absoluta; Fr=Frecuencia Relativa; IVI=Índice Valor de Importancia

Las especies dominantes son: Handroanthus chrysanthus 23,26 \% (3,78 $\left.\mathrm{m}^{2}\right)$, Ceiba trichistandra 12,91\% (2,10 $\left.\mathrm{m}^{2}\right)$, Eriotheca ruizii 11,58\% $\left(1,88 \mathrm{~m}^{2}\right)$, Cochlospermum vitifolium $9,33 \%\left(1,80 \mathrm{~m}^{2}\right)$. Las especies con menor dominancia son: Allophylus sp. 0,04 $\%\left(0,01 \mathrm{~m}^{2}\right)$, Senna mollissima, Acacia macracantha con 0,21\% (0,03 m²); y, Croton sp., Maclura tinctoria $0,01 \%\left(0,002 \mathrm{~m}^{2}\right)$

Las especies con mayor IVI en El Tabanco por su densidad, frecuencia y dominancia relativa son: Handroanthus chrysanthus (15,57 \%), Simira ecuadorensis (9,93\%), Citharexylum gentryi (7,06\%); Eriotheca ruizii (5,67), Chloroleucon mangense $(5,66 \%)$, Celtis loxensis (5,31\%) у Cochlospermum vitifolium (5,16\%).

\section{Parámetros estructurales del estrato arbustivo}

En el estrato arbustivo se registraron 162 individuos pertenecientes a nueve parcelas de $225 \mathrm{~m}^{2}$, que pertenecen a 7 especies de 6 géneros dentro de 6 familias. En la Tabla 2, se presenta los parámetros de los individuos registrados. 
Tabla 2. Parámetros estructurales de las especies del estrato arbustivo de la parcela del bosque seco del sector El Tabanco, Mangahurco, Zapotillo.

\begin{tabular}{|c|c|c|c|c|c|c|c|}
\hline $\mathbf{N}^{\mathbf{o}}$ & Familia & Nombre Científico & $\begin{array}{l}\mathrm{N}^{\circ} \\
\text { Ind. }\end{array}$ & $\begin{array}{c}\text { D } \\
\text { (Ind./ha) }\end{array}$ & $\begin{array}{l}\text { DR } \\
(\%)\end{array}$ & $\begin{array}{l}\text { Fr } \\
(\%)\end{array}$ & IVI \\
\hline 1 & Euphorbiaceae & Croton sp. 1 & 73 & 3244 & 45,06 & 32 & 77,06 \\
\hline 2 & Apocinaceae & Rauvolfia tetraphyylla $\mathrm{L}$. & 26 & 1156 & 16,05 & 29 & 45,05 \\
\hline 3 & Asteraceae & $\begin{array}{l}\text { Dendrophorbium lloense (Hieron. ex } \\
\text { Sodiro) C. Jeffrey }\end{array}$ & 51 & 2267 & 31,48 & 14 & 45,48 \\
\hline 4 & Nyctaginaceae & Bougainvillea peruviana Bonpl. & 1 & 44 & 0,62 & 4 & 4,62 \\
\hline 5 & Rubiaceae & Duroia sp. & 4 & 178 & 2,47 & 7 & 9,47 \\
\hline 7 & Asteraceae & Cordia macrocephafa (Desv.) Kunth & 3 & 133 & 1,85 & 7 & 8,85 \\
\hline 8 & Euphorbiaceae & Croton sp. 2 & 4 & 178 & 2,47 & 4 & 6,47 \\
\hline \multicolumn{3}{|c|}{ Total } & 162 & 7200 & 100 & 100 & 200 \\
\hline
\end{tabular}

$D=$ Densidad $;$ DR = Densidad Relativa $; F r=$ Frecuencia Relativa $; I V I=$ Índice Valor de Importancia

En el estrato arbustivo, las especies más densas son: Croton sp. 1. con 3244 individuos/ha (45,06 \%) y Rauvolfia tetraphylla 2256 individuos/ha (16,05\%). La especie con menor densidad es Bougainvillea peruviana con 44 individuos/ha (0,62\%).

Las especies con mayor frecuencia relativa son: Croton sp. 1 con el $32 \%$, Rauvolfia tetraphylla con el $29 \%$ y Dendrophorbium lloense con el $14 \%$, mientras que Bougainvillea peruviana y Crotón sp. 2, presentan el $4 \%$.

Las especies con mayor IVI son: Croton sp. 1., Rauvolfia tetraphylla y Dendrophorbium lloense, debido a su abundancia numérica.

\section{Parámetros estructurales del estrato herbáceo}

Se contabilizaron 236 individuos en $9 \mathrm{~m}^{2}$, pertenecientes a 18 especies, 18 géneros de 8 familias. En la Tabla 3, se presenta los parámetros estructurales de las especies de hierbas registradas en época lluviosa con su densidad, densidad relativa y frecuencia.

Tabla 3. Parámetros estructurales de las especies del estrato herbáceo del bosque de la parcela en el bosque seco de El Tabanco, Mangahurco, Zapotillo.

\begin{tabular}{|c|l|c|c|c|c|c|}
\hline $\mathbf{N}^{\mathbf{0}}$ & \multicolumn{1}{|c|}{ Nombre Científico } & $\begin{array}{c}\text { No. } \\
\text { Ind. }\end{array}$ & $\begin{array}{c}\text { D } \\
\text { (Ind./ha) }\end{array}$ & $\begin{array}{c}\text { DR } \\
(\boldsymbol{\%})\end{array}$ & $\begin{array}{c}\text { Fr } \\
(\boldsymbol{\%})\end{array}$ & $\begin{array}{c}\text { IVI } \\
\mathbf{\%}\end{array}$ \\
\hline 1 & Bastardia bivalvis (Cav.) Kunth & 21 & 23333 & 8,90 & 8,16 & 17,06 \\
\hline 2 & Desmodium cf. Procumbens (Mill) Hitchc & 5 & 5556 & 2,12 & 4,08 & 6,20 \\
\hline 3 & Bidens pilosa L. & 46 & 51111 & 19,49 & 14,29 & 33,78 \\
\hline 4 & Hyptis sp & 7 & 7778 & 2,97 & 4,08 & 7,05 \\
\hline
\end{tabular}




\begin{tabular}{|c|l|c|c|c|c|c|}
\hline 5 & $\begin{array}{l}\text { Chromolaena roseorum (b. l. Rob.) R. M. King \& } \\
\text { H. Rob }\end{array}$ & 17 & 18889 & 7,20 & 8,16 & 15,36 \\
\hline 6 & Pavonia sepium A. St. Hill & 5 & 5556 & 2,12 & 2,04 & 4,16 \\
\hline 7 & Convolvulus sp. & 2 & 2222 & 0,85 & 2,04 & 2,89 \\
\hline 8 & Rhynchosia sp. & 1 & 1111 & 0,42 & 2,04 & 2,46 \\
\hline 9 & Viguieria sp & 1 & 1111 & 0,42 & 2,04 & 2,46 \\
\hline 10 & Tradescantia sp. & 34 & 37778 & 14,41 & 10,20 & 24,61 \\
\hline 11 & Vastardia sp. & 12 & 13333 & 5,08 & 4,08 & 9,16 \\
\hline 12 & Dicliptera paposana Phil. & 45 & 50000 & 19,07 & 12,24 & 31,31 \\
\hline 13 & Fiebrigiella graciles Harms & 1 & 1111 & 0,42 & 2,04 & 2,46 \\
\hline 14 & Bidens sp. & 28 & 31111 & 11,86 & 12,24 & 24,10 \\
\hline 15 & Achirantes sp. & 2 & 2222 & 0,85 & 2,04 & 2,89 \\
\hline 16 & Browallia americana L. & 6 & 6667 & 2,54 & 4,08 & 6,62 \\
\hline 17 & Ipomoea sp. & 1 & 1111 & 0,42 & 2,04 & 2,46 \\
\hline 18 & Abutilon mollissimum (Cav.) Sweet & $\mathbf{2 3 6}$ & $\mathbf{2 6 2 2 2 2}$ & $\mathbf{1 0 0}$ & $\mathbf{1 0 0}$ & $\mathbf{2 0 0}$ \\
\hline & Total & 2222 & 0,85 & 4,08 & 4,93 \\
\hline
\end{tabular}

$D=$ Densidad; DR= Densidad Relativa; Fr=Frecuencia Relativa $;$ IVI = Índice Valor de Importancia.

Las especies más densas son: Bidens pilosa con 51111 individuos/ha (19,49 \%), Dicliptera paposana, con 50000 individuos/ha (19,07\%) y Tradescantia sp. con 37778 individuos/ha (14,41\%) y Bidens sp. con 31111 individuos/ha (11,86\%).

Las especies más frecuentes son: Bidens pilosa con 14,29 \%, Dicliptera paposana y Bidens sp. con el 12,24 \% cada una. Y las especies menos frecuentes son: Pavonia sepium, Convolvulus sp., Rhynchosia sp., Viguieria sp., Fiebrigiella sp., Achirantes sp. y Abutilon mollissimum con $2,04 \%$ cada una.

Las especies con mayor IVI son: Bidens pilosa (33,78), Dicliptera paposana $(31,31)$, Tradescantia sp. $(24,61)$ y Bidens sp. $(24,10)$.

\section{Parámetros estructurales de las epífitas vasculares}

Se contabilizaron 736 individuos en 25 árboles estudiados, que pertenecen a 4 especies dentro de 4 géneros y 2 familias. Los parámetros de las epífitas vasculares se presentan en la Tabla 4, con su densidad, densidad relativa y frecuencia. 
Tabla 4. Parámetros estructurales de epífitas vasculares de la parcela permanente en el bosque seco de El Tabanco, Mangahurco, Zapotillo.

\begin{tabular}{|c|l|l|c|c|c|c|}
\hline $\mathbf{N}^{\mathbf{0}}$ & \multicolumn{1}{|c|}{ Familia } & \multicolumn{1}{|c|}{ Nombre Científico } & $\begin{array}{c}\text { D } \\
\text { (Ind./ha) }\end{array}$ & $\begin{array}{c}\text { DR } \\
(\%)\end{array}$ & $\begin{array}{c}\mathbf{F} \\
\mathbf{a b}\end{array}$ & $\begin{array}{c}\text { Fr } \\
(\boldsymbol{\%})\end{array}$ \\
\hline 1 & Bromeliaceae & Vriesea espinosae (L. B. Sm) Gilmartin & 600 & 81,52 & 15 & 34,09 \\
\hline 2 & Orchidaceae & $\begin{array}{l}\text { Zelencoa onusta (Lindl.) M. W. Chase \& N. H. } \\
\text { Williams }\end{array}$ & 44 & 5,98 & 15 & 34,09 \\
\hline 3 & Bromeliaceae & Tillandsia usneoides (L.) L. & 78 & 10,60 & 9 & 20,45 \\
\hline 4 & Bromeliaceae & Tillandsia spiralipetala Gouda & 13 & 1,77 & 4 & 9,09 \\
\hline 5 & Orchidaceae & Catasetum sp. & 1 & 0,14 & 1 & 2,27 \\
\hline \multicolumn{2}{|l|}{ Total } & $\mathbf{7 3 6}$ & $\mathbf{1 0 0 , 0 0}$ & $\mathbf{4 4}$ & $\mathbf{1 0 0}$ \\
\hline
\end{tabular}

DR= Densidad Relativa; Fr=Frecuencia Relativa.

La mayor densidad poseen: Vriesea espinosae con 600 individuos/25 árboles $(81,52 \%)$ y Zelencoa onusta con 44 individuos/25 árboles (5,98 \%). Catasetum sp. es la menos densa con 1 individuo/25 árboles $(0,14 \%)$.

Las epífitas con mayor frecuencia en los árboles hospederos son: Vriesea espinosae con $34,09 \%$ y Zelencoa onusta con 34,09 \%. La especie menos frecuente es Catasetum sp. $(2,27 \%)$.

\section{Diversidad alfa del bosque}

El índice de diversidad de Shannon, muestra que, para los individuos mayores a $5 \mathrm{~cm}$ de DAP, la diversidad tiene un valor de 0,38. Para el estrato arbustivo un valor de 0,26 y en el estrato herbáceo 0,42 , que significa diversidad media, baja y media respectivamente.

\section{Estructura diamétrica del bosque.}

Se diferencian tres estratos, sobresaliendo en el estrato superior: Cochlospermum vitifolium, Eriotheca ruizii, Ceiba trichistandra, Machaerium millei, Terminalia valverdae y Handroanthus chrysanthus, en el estrato codominante Simira ecuadorensis, Chloroleucon mangense, Celtis loxensis, Caesalpina glabrata y Geoffroea spinosa y en el estrato suprimido Celtis loxensis, Pisonia aculeata y Cynophylla sclerophylla.

Se registran $1117 \mathrm{ind} / \mathrm{ha}$ mayores a $5 \mathrm{~cm}$ de $\mathrm{D}_{1,30 \mathrm{~m}}$, que reportan un área basal de 16,99 $\mathrm{m}^{2} /$ ha y volumen de $61,11 \mathrm{~m}^{3} / \mathrm{ha}$ (Tabla 5); éstos se agrupan en 10 clases diamétricas, siendo las tres primeras las más abundantes $(96,06 \%)$ con diámetros menores; esta característica hace que la distribución diamétrica tenga la forma de "J" invertida (Figura 2). 
Tabla 5. Datos dasométricos y volumétricos por clases diamétricas de la parcela permanente del bosques seco del sector “El Tabanco”, Mangahurco, Zapotillo, Ecuador.

\begin{tabular}{|c|c|c|c|c|c|c|c|}
\hline Clase & $\begin{array}{c}\text { Rangos } \\
\text { diamétricos } \\
\mathbf{( c m})\end{array}$ & $\begin{array}{c}\text { Número de } \\
\text { individuos }\end{array}$ & $\begin{array}{c}\text { DAP } \\
\text { promedio } \\
\mathbf{m}\end{array}$ & $\begin{array}{c}\text { Altura } \\
\text { promedio } \\
(\mathbf{m})\end{array}$ & $\begin{array}{c}\text { Área } \\
\text { basal/ha } \\
\left(\mathbf{m}^{\mathbf{2} / \mathbf{h a})}\right.\end{array}$ & $\begin{array}{c}\text { Factor } \\
\text { promedio }(\mathbf{f})\end{array}$ & $\begin{array}{c}\text { Volume } \\
\mathbf{n} \text { total } \\
\mathbf{m}^{\mathbf{3}} \mathbf{h a}\end{array}$ \\
\hline I & $5-12,81$ & 860 & 0,09 & 5,75 & 3,95 & 0,4123 & 8,04 \\
\hline II & $12,82-20,63$ & 153 & 0,17 & 9,50 & 4,06 & 0,4123 & 10,01 \\
\hline III & $20,64-28,45$ & 60 & 0,24 & 12,25 & 2,71 & 0,4123 & 11,38 \\
\hline IV & $28,46-36,27$ & 23 & 0,32 & 13,25 & 1,87 & 0,4123 & 7,33 \\
\hline V & $36,28-44,09$ & 11 & 0,40 & 11,80 & 1,34 & 0,4123 & 6,35 \\
\hline VI & $44,10-51,91$ & 3 & 0,47 & 13,55 & 0,51 & 0,4123 & 2,74 \\
\hline VII & $51,92-59,73$ & 3 & 0,54 & 15,6 & 0,70 & 0,4123 & 4,47 \\
\hline VIII & $59,74-67,55$ & 0 & 0,00 & 0 & 0,00 & 0,4123 & 0,00 \\
\hline IX & $67,56-75,81$ & 1 & 0,69 & 10,8 & 0,38 & 0,4123 & 1,68 \\
\hline X & $75,82-83,63$ & 3 & 0,80 & 14,80 & 1,49 & 0,4123 & 9,10 \\
\hline Total & & $\mathbf{1 1 1 7}$ & & $\mathbf{1 6 , 9 9}$ & & & $\mathbf{6 1 , 1 1}$ \\
\hline
\end{tabular}

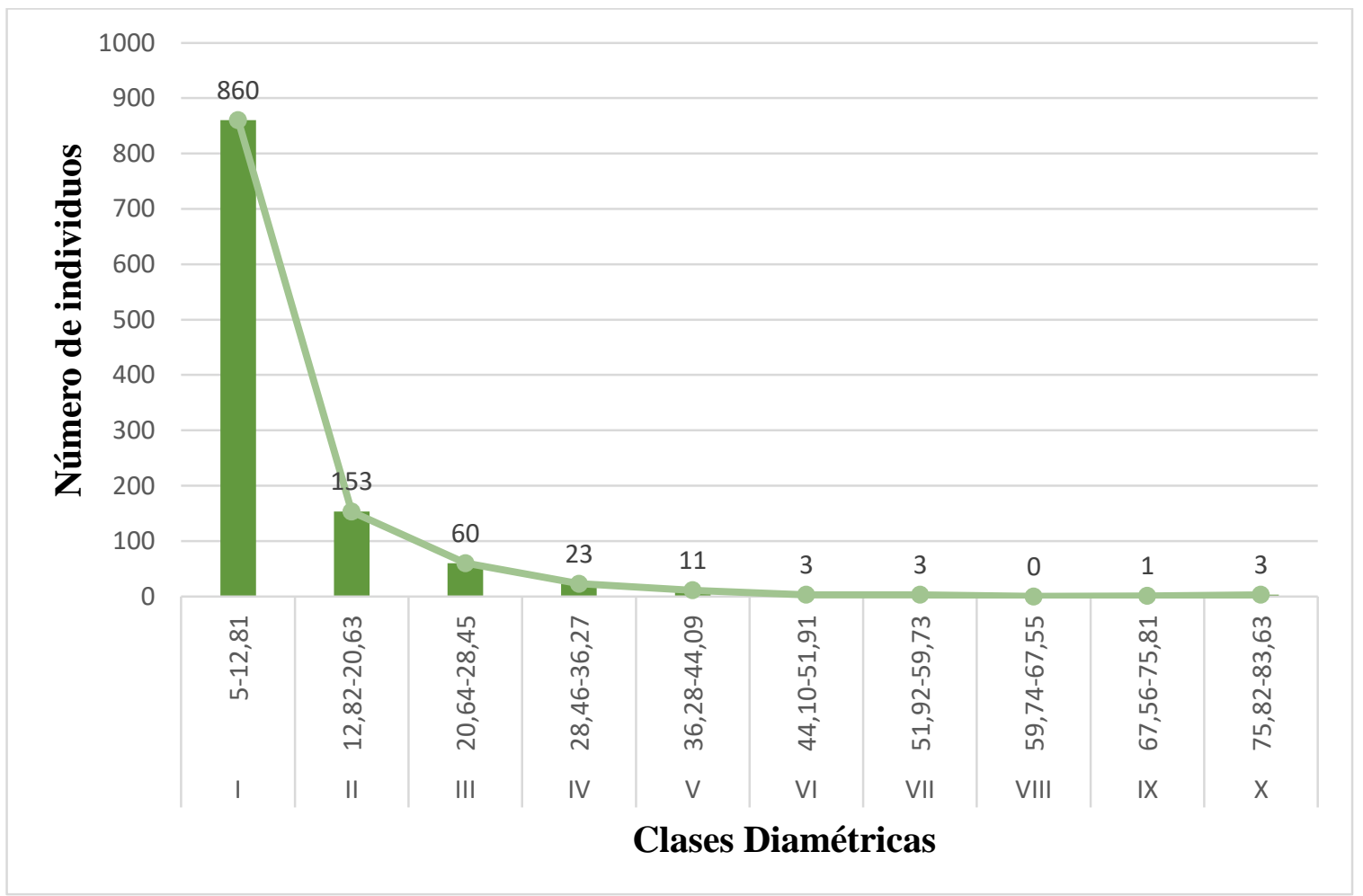

Figura 2. Distribución diamétrica del bosque seco de Mangahurco, Zapotillo, Loja. Endemismo 
Se reporta una especie endémica para Ecuador, desde este bosque, Citharexylum gentryi, y nueve especies como endémicas de la Región Tumbesina que comparten Ecuador y Perú. En la Tabla 6 se presentan la lista de especies endémicas compartidas.

Tabla 6. Especies endémicas registradas en la parcela permanente "El Tabanco" y que son compartidas entre Ecuador-Perú.

\begin{tabular}{|l|l|l|l|l|}
\hline $\mathbf{N}^{\mathbf{0}}$ & \multicolumn{1}{|c|}{ Familia } & \multicolumn{1}{|c|}{ Nombre Científico } & \multicolumn{1}{|c|}{$\begin{array}{c}\text { Nombre } \\
\text { Común }\end{array}$} & \multicolumn{1}{|c|}{ Endemismo } \\
\hline 1 & Fabaceae & Erythrina velutina Willd. & Porotillo & Ecuador y Perú \\
\hline 2 & Malvaceae & Ceiba trichistandra (A. Gray) Bakh. & Ceibo & Ecuador y Perú \\
\hline 3 & Combretaceae & Terminalia valverdae A.H. Gentry & Guarapo & Ecuador y Perú \\
\hline 4 & Erythroxylaceae & Erythroxylum glaucum O.E. Schulz & Negro & Ecuador y Perú \\
\hline 5 & Fabaceae & Albizia multiflora (Kunth.) Barneby & Angolo & Ecuador y Perú \\
\hline 6 & Rubiaceae & Simira ecuadorensis (Standl.) Steyerm. & Guápala & Ecuador y Perú \\
\hline 7 & Ulmaceae & Celtis loxensis Cc. Berg & Palo blanco & Ecuador y Perú \\
\hline 8 & Bignoniaceae & $\begin{array}{l}\text { Handroanthus billbergii (Bureau \& K. } \\
\text { Schum) S. O. Grose. }\end{array}$ & $\begin{array}{l}\text { Guayacán } \\
\text { madero }\end{array}$ & Ecuador y Perú \\
\hline 9 & Moraceae & Ficus jacobii Vázq. Avila & Mata palo & Ecuador y Perú \\
\hline
\end{tabular}

\section{DISCUSIÓN}

\section{Composición florística}

Los datos demuestran que en la parcela permanente del bosque El Tabanco la diversidad es mayor comparando con estudios realizados en el bosque seco de la Reserva La Ceiba por Aguirre et al (2014) que reconoció 28 especies de árboles, Granda y Guamán (2006) que contabilizan 33 especies de árboles y Bustamante (2009) registró 24 especies arbóreas. En el estrato arbustivo la especie con mayor densidad relativa es Croton sp.1 con $3244 \mathrm{ind} / \mathrm{ha}$, que representa el 45,06 \%, este resultado es igual a lo reportado por Bustamante (2009) con 45,16 \%; Aguirre et al. (2014) en su investigación en La Ceiba presenta también a Croton sp. como la especie con mayor densidad relativa con 45,51\%, este resultado podría ser, debido a que el bosque se está recuperando de intervenciones pasadas; y al buena regeneración natural de algunas especies como Simira ecuadorensis y Handroanthus chrysanthus.

\section{Diversidad alfa del bosque}


La diversidad florística de la parcela de bosque seco El Tabanco según el índice de Shannon tiene un valor de 0,38 del estrato arbóreo, que significa una diversidad media, mientras que Granda y Guamán (2006) en su estudio registran la misma diversidad con un valor de 0,42. Además, Aguirre et al. (2014) determinó una diversidad alfa baja de 0,31. En el estrato arbustivo la diversidad de Shannon tiene un valor de 0,26 que significa que la diversidad del bosque para este estrato es baja y en el estrato herbáceo la diversidad es media con un valor de 0,42 .

\section{Diversidad de familia}

Las familias más diversas en la parcela del bosque seco El Tabanco son: Fabaceae, Asteraceae, Malvaceae y Rubiaceae datos que concuerdan con lo reportado por Gentry (1995), Aguirre et al. (2001), Granda y Guamán (2006), Aguirre et al. (2014), Bustamante (2009), Aguirre y Kvist (2009) y Aguirre (2013) que también consideran a las Fabaceae y Malvaceae como el grupo mejor representado en los bosques secos del Ecuador.

\section{Parámetros estructurales de la vegetación}

Handroanthus chrysanthus y Ceiba trichistandra son las especies dominantes, debido a su abundancia y mayor área basal, también Aguirre et al. (2006), Aguirre (2013), Aguirre et al. (2013), Aguirre et al. (2014) afirman que Ceiba trichistandra, Handroanthus chrysanthus, Eriotheca ruizii y Simira ecuadorensis tiene mayor importancia ecológica y que son las especies típicas y características de los bosques secos de Ecuador y Perú, en comparación con otros bosques secos del mundo.

\section{Parámetros dasométricos de la vegetación.}

El bosque seco El Tabanco tiene un área basal de 16,99 m²/ha y volumen de $61,11 \mathrm{~m}^{3} / \mathrm{ha}$; este resultado es menor a lo reportado por Granda y Guamán (2006) que reportan 23,45 $\mathrm{m}^{2} /$ ha de área basal y $111,78 \mathrm{~m}^{3} / \mathrm{ha}$ de volumen, mientras que Aguirre et al. (2014) registró $26,73 \mathrm{~m}^{2} /$ ha y volumen de $169,41 \mathrm{~m}^{3} / \mathrm{ha}$, Aguirre et al. (2001), Aguirre y Kvist (2009) y Aguirre (2013) en el sector La Ceiba que registra un valor de 20,33 $\mathrm{m}^{2} / \mathrm{ha}$, la diferencia se debe al mayor número de individuos/ha de Simira ecuadorensis, Handroanthus chrysanthus y Citharexylum gentryi y, a la presencia de pocos árboles con mayor área basal y alturas, especialmente de Ceiba trichistandra, Eriotheca ruizii, Terminalia valverdeae, Erythrina velutina y Cochlospermum vitifolium

\section{Estructura diamétrica del bosque}


Según Granda y Guamán (2006), Bustamante (2009), Aguirre et al. (2013) y Aguirre et al. (2014) los árboles que conforman este tipo de bosque seco son delgados y escasos individuos de gran tamaño se encuentran dispersos, esta característica es típica de los bosques intervenidos que han sufrido alteración por la intervención humana, esto se confirma en este estudio, indicando que la mayor cantidad de individuos se agrupan en las tres primeras clases diamétricas entre 5 y $28 \mathrm{~cm}$ de DAP, lo cual es ratificado por Lamprecht (1990) y Aguirre (2013) que indica que la distribución diamétrica en bosques nativos jóvenes en procesos de recuperación y presentan una tendencia de "J" invertida.

\section{Endemismo}

El bosque seco del occidente de la provincia de Loja es considerado de gran importancia biológica, debido a los altos niveles de endemismo de especies especialmente de fauna, se reporta una especie endémica de Ecuador y nueve compartidas en la zona endemismo Tumbesino que corresponden a Ecuador y Perú, este ecosistema se encuentra en zonas pobladas y soporta amenazas por las actividades antrópicas, aseveración que es corroborada por Aguirre et al. (2006), Aguirre y Kvist (2009) y Aguirre (2013).

\section{CONCLUSIONES}

- La composición florística del bosque es de 61 especies, 32 especies leñosas mayores o iguales a $5 \mathrm{~cm}$ de DAP, 7 arbustos, 18 hierbas y 4 epífitas. Las especies ecológicamente más importantes del bosque El Tabanco son: Handroanthus chrysanthus (Jacq.) S. O. Grose, Simira ecuadorensis (Standl.) Steyerm., Citharexylum gentryi Moldenke y Eriotheca ruizii (K. Schum.) A. Robyns.

- El área basal de las especies de la parcela es $16,99 \mathrm{~m}^{2} /$ ha y el volumen es $61,11 \mathrm{~m} 3 / \mathrm{ha}$; las especies que más aportan son: Ceiba trichistandra, Handroanthus chrysanthus, Cochlospermum vitifolium y Eriotheca ruizii.

- Las tres primeras clases diamétricas agrupan la mayor cantidad de árboles evaluados, que determina una distribución diamétrica con una tendencia a "J" invertida, indicando que el bosque seco de El Tabanco es un bosque en proceso de recuperación.

- La estructura vertical del bosque de El Tabanco, destaca tres estratos bien definidos: dominante, codominado y suprimido, Cochlospermum vitifolium, Eriotheca ruizii, Ceiba trichistandra, Machaerium millei, Terminalia valverdae, y Handroanthus 
chrysanthus son las especies que dominan y aparecen como típicas y características de estos bosques.

- El bosque El Tabanco es el hábitat de 10 especies endémicas, una exclusiva de Ecuador y nueve endémicas de la Región Tumbesina, tratándose de un endemismo compartido entre Ecuador y Perú.

\section{BIBLIOGRAFÍA}

Aguirre, Z.; Cueva, E.; Merino, B.; Quizhpe, W.; Valverde, A. (2001). Evaluación ecológica rápida de la vegetación en los bosques secos La Ceiba y Cordillera Arañitas, provincia de Loja. Ecuador. En: Vázquez, M. A., Larrea, M., Suarez, L. Y Ojeda, P. (Eds.). Biodiversidad en los bosques secos del suroccidente de la provincia de Loja: un reporte de las evaluaciones ecológicas y socioeconómicas rápidas (pp. 16 - 35). EcoCiencia, Ministerio del Ambiente, Herbario LOJA y Proyecto Bosque Seco.

Aguirre, Z. Linares, R. y Kvist, L. (2006). Especies leñosas y formaciones vegetales en los bosques estacionalmente secos de Ecuador y Perú. Revista Arnaldoa, 13 (2): 324-350.

Aguirre, Z., Kvist, L. y Sánchez, O. (2006). Bosques secos en Ecuador y su diversidad. En Moraes, M. Ollgard, B. Kvist, L. Borchseniu, F, y Baslev, H. (Eds.). Botánica Economica de los Andes Centrales (pp. 162-167). Universidad Mayor San Andrés, La Paz Bolivia.

Aguirre, Z. y Kvist, L. (2009). Composición florística y estructura de bosques estacionalmente secos en el sur-occidental de Ecuador, provincia de Loja, municipios de Macara y Zapotillo. Revista Arnaldoa 16(2): 87 - 99.

Aguirre, Z. (2013). Estructura del bosque seco de la provincia de Loja y sus productos forestales no maderables: caso de estudio Macará. (Tesis de postgrado, Universidad de Pinar de Río). Pinar del Rio, Cuba.

Aguirre-Mendoza, Z.; Betancourt-Figueras, Y.; Geada-López, G. (2013). Composición florística, estructura de los bosques secos y su gestión para el desarrollo de la provincia de Loja, Ecuador. Revista Avances. 15(2): 134-146.

Aguirre-Mendoza Z., Buri Sivisaca D., Betancourt Y. y Geada López G. (2014). Composición florística, estructura y endemismo en una parcela permanente de 
bosque seco en Zapotillo, Provincia de Loja, Ecuador. Revista Arnaldoa, 21(1): $165-178$.

Aguirre Z. (2019). Métodos para medir la Biodiversidad. Primera Edición. Universidad Nacional de Loja. Loja, Ecuador. ISBN: 978-9942-36-127-1

Bustamante, T. (2009). Composición florística, estructura y endemismo en el bosque seco de la Reserva Natural Laipuna, Macará, Loja. (Trabajo de grado de Ingeniero Forestal, Universidad Nacional de Loja). Loja, Ecuador.

Cerón, C. (2003). Manual de botánica, sistemática, etnobotánica y métodos de estudio. Herbario “Alfredo Paredes” QAP. Escuela de Biología de la Universidad Central del Ecuador. Quito-Ecuador.

Granda, V.; Guamán, S. (2006). Composición florística, estructura, endemismo y etnobotánica de los bosques secos Algodonal y la Ceiba en los cantones Macará y Zapotillo de la provincia de Loja. (Trabajo de grado de Ingeniería Forestal, Universidad Nacional de Loja). Loja-Ecuador.

Klitgard, B.; Lozano. P.; Aguirre, Z.; Merino, B.; Aguirre, N.; Delgado, T.; y Elizalde, F. (1990). Composición florística y estructural del Bosque Petrificado de Puyango. Loja-Ecuador. En: Estudios Botánicos en el Sur del Ecuador $N^{\circ} 3$. Universidad Nacional de Loja, departamento de Botánica y Ecología. Ecuador.

Herbario Loja, CINFA, UNISIG. (2001). Zonificación y delimitación de tipos de vegetación del bosque seco del sur occidente de la provincia de Loja. Proyecto Bosque Seco. Loja-Ecuador.

Lamprecht, H. (1990). Silvicultura en los trópicos. Trad. Antonio Carrillo, GTZ, Alemania.

Linares-Palomino, R. (2004). Los bosques tropicales estacionalmente secos: I. El concepto de los bosques secos en el Perú. Revista Arnolda 11(1), 85-102.

Ministerio del Ambiente del Ecuador. (2013). Sistema de clasificación de los ecosistemas del Ecuador continental. Subsecretaría de Patrimonio Natural. Quito. Ministerio del Ambiente. http://areasprotegidas.ambiente.gob.ec/es/content/bosques-protectores

Paladines, R. (2003). Propuesta de conservación del bosque seco en el sur del Ecuador. Fundación Científica San Francisco. 
http//www.lyonia.org/Archives/Lyonia\%204(2)\%202003(103230)/Paladine s\%20P.,\%20R.\%\%3B\%20Lyonia\%204(2)\%202003(183286).pdf

Pennington, R. T.; D. E. Prado \& C. A. Pendry. (2000). Neotropical seasonally dry forests and quaternary vegetation changes. Journal of Biogeography, 27, 261-273.

Phillips, O., Baker, T., Feldpausch, T. Y Brienen, R., (2016). Manual de Campo para el Establecimiento y la Remedición de Parcelas [en línea]. Perú: RAINFOR. http://www.rainfor.org/upload/ManualsSpanish/Manual/RAINFOR_field_m anu al_version2016_ES.pdf. 
ANEXO

Anexo 1. Especies de plantas vasculares inventariadas en la parcela permanente "El Tabanco",

Mangahurco, Ecuador.

\begin{tabular}{|c|c|c|}
\hline Nombre Científico & Nombre Común & $\begin{array}{l}\text { Hábito de } \\
\text { crecimiento }\end{array}$ \\
\hline \multicolumn{3}{|l|}{ Amaranthaceae } \\
\hline Dicliptera paposana Phil. & Monte malo & $\mathrm{H}$ \\
\hline Achirantes sp. & Monte lanudo & $\mathrm{H}$ \\
\hline \multicolumn{3}{|l|}{ Bignoniaceae } \\
\hline Handroanthus chrysanthus (Jacq.) Nicholson & Guayacán oreja de León & $\mathrm{A}$ \\
\hline Handroanthus billbergii (Bureau \& K. Schum) Standl. & Guayacán madero & $\mathrm{A}$ \\
\hline \multicolumn{3}{|l|}{ Apocinaceae } \\
\hline Rauvolfia tetraphyylla $\mathrm{L}$. & Guaruz & $\mathrm{Ar}$ \\
\hline \multicolumn{3}{|l|}{ Asteraceae } \\
\hline Dendrophorbium lloense (Hieron. ex Sodiro) C.Jeffrey & Tabaco cimarrón & $\mathrm{Ar}$ \\
\hline Cordia macrocephafa (Desv.) Kunth & Palo negro & $\mathrm{Ar}$ \\
\hline Bidens pilosa $\mathrm{L}$. & Buchingue & $\mathrm{H}$ \\
\hline Bidens sp. & Manzanillo de vaca & $\mathrm{H}$ \\
\hline Chromolaena roseorum (B. 1. Rob.) R. M. King \& H. Rob & Monte blanco & $\mathrm{H}$ \\
\hline Viguieria sp. & Tarapillo & $\mathrm{H}$ \\
\hline \multicolumn{3}{|l|}{ Bixaceae } \\
\hline Cochlospermum vitifolium (Willd.) Spreng. & Polo-polo & $\mathrm{A}$ \\
\hline \multicolumn{3}{|l|}{ Bromeliaceae } \\
\hline Vriesea espinosae (L. B. Sm) Gilmartin & Achupalla & E \\
\hline Tillandsia spiralipetala Gouda & Musgo & E \\
\hline Tillandsia usneoides (L.) L. & Barba de San Pedro & $\mathrm{E}$ \\
\hline \multicolumn{3}{|l|}{ Bursearceae } \\
\hline Bursera graveolens (Kunth) Triana \& Planch & Palo santo & $\mathrm{A}$ \\
\hline \multicolumn{3}{|l|}{ Capparaceae } \\
\hline Cynophylla sclerophylla (H. H. lltis \& X. Cornejo) & Limoncillo & $\mathrm{A}$ \\
\hline \multicolumn{3}{|l|}{ Combretaceae } \\
\hline Terminalia valverdeae A.H. Gentry & Guarapo & $\mathrm{A}$ \\
\hline \multicolumn{3}{|l|}{ Convolvulaceae } \\
\hline Convolvulus sp. & Carriguela lanuda & $\mathrm{H}$ \\
\hline Ipomoea sp. & Camotillo & $\mathrm{H}$ \\
\hline \multicolumn{3}{|l|}{ Conmelinaceae } \\
\hline Tradescantia sp. & Carricillo & $\mathrm{H}$ \\
\hline \multicolumn{3}{|l|}{ Euphorbiaceae } \\
\hline Croton sp. 1 & Moshquero & $\mathrm{A}$ \\
\hline Croton sp. 2 & Moshquera & $\mathrm{Ar}$ \\
\hline Croton sp. 3 & Moshquero macho & $\mathrm{Ar}$ \\
\hline
\end{tabular}




\begin{tabular}{|c|c|c|}
\hline Erythroxylaceae & & \\
\hline Erythroxylum glaucum O.E. Schulz & Negrito & $\mathrm{A}$ \\
\hline \multicolumn{3}{|l|}{ Fabaceae } \\
\hline Acacia macracantha Humb. \& Bonpl. Ex Willd. & Faique & A \\
\hline Albizia multiflora (Kunth) Barneby \& J.W. Grimes & Angolo & $\mathrm{A}$ \\
\hline Bauhinia aculeata $L$. & Uña de coche & $\mathrm{Ar}$ \\
\hline Calliandra taxifolia (Kunth.) Benth. & Barba de chivato & $\mathrm{Ar}$ \\
\hline Caesalpina glabrata Kunth. & Charan negro & $\mathrm{A}$ \\
\hline Chloroleucon mangense (Jacq.) Britton \& Rose & Charan Serrano & $\mathrm{A}$ \\
\hline Desmodium cf. procumbens (Mill) Hitchc & Miñate & $\mathrm{H}$ \\
\hline Erythrina velutina Willd & Porotillo & A \\
\hline Fiebrigiella graciles Harms & Culantrillo & $\mathrm{H}$ \\
\hline Geoffroea spinosa Jacq. & Almendro & $\mathrm{A}$ \\
\hline Leucaena trichodes (Jacq.) Benth. & Chapra & $\mathrm{A}$ \\
\hline Machaerium millei Standl. & Chicho & $\mathrm{A}$ \\
\hline Piptadenia flava (Spreng. Ex DC.) Benth. & Serrillo & $\mathrm{A}$ \\
\hline Rhynchosia sp. & Sarandajilla & $\mathrm{H}$ \\
\hline $\begin{array}{l}\text { Senna mollissima (Humb. \& Bonpl. Ex Willd.) H.S. Irwin \& } \\
\text { Barnaby. }\end{array}$ & Vainillo & A \\
\hline \multicolumn{3}{|l|}{ Lamiaceae } \\
\hline Hyptis sp & Hoja picuda & $\mathrm{H}$ \\
\hline \multicolumn{3}{|l|}{ Malvaceae } \\
\hline Abutilon mollissimum (Cav.) Sweet & Varilla & $\mathrm{H}$ \\
\hline Ceiba trichistandra (A. Gray) Bakh. & Ceibo & A \\
\hline Eriotheca ruizii (K. Schum.) A. Robyns & Pasallo & A \\
\hline Pavonia sepium A. St. Hill & Sierra & $\mathrm{H}$ \\
\hline Vastardia sp. & & $\mathrm{H}$ \\
\hline \multicolumn{3}{|l|}{ Meliaceae } \\
\hline Cedrela odorata $\mathrm{L}$. & Cedro & $\mathrm{A}$ \\
\hline \multicolumn{3}{|l|}{ Moraceae } \\
\hline Ficus jacobii Vázq. Avila & Mata palo & A \\
\hline Maclura tinctoria L. Steud & lechoso & $\mathrm{A}$ \\
\hline \multicolumn{3}{|l|}{ Nyctaginaceae } \\
\hline Pisonia aculeata $\mathrm{L}$. & Pego-pego & $\mathrm{A}$ \\
\hline Bougainvillea peruviana Bonpl. & Papelillo & $\mathrm{Ar}$ \\
\hline \multicolumn{3}{|l|}{ Orchidaceae } \\
\hline Zelencoa onusta (Lindl.) M. W. Chase \& N. H. Williams & Achupalla gallito & $\mathrm{E}$ \\
\hline Catasetum sp. & Orquídea & $\mathrm{E}$ \\
\hline \multicolumn{3}{|l|}{ Rhamnacae } \\
\hline Ziziphus thyrsiflora Benth. & Ébano & $\mathrm{A}$ \\
\hline \multicolumn{3}{|l|}{ Rubiaceae } \\
\hline Simira ecuadorensis (Standl.) Steger & Guápala & A \\
\hline
\end{tabular}


Bosque seco de

\begin{tabular}{|l|l|c|} 
Randia armata $(\mathrm{SW})$. DC. & Churipingo & $\mathrm{A}$ \\
\hline Duroiasp. & Tumba Jinete & $\mathrm{Ar}$ \\
\hline Solanaceae & & $\mathrm{H}$ \\
\hline Browallia americana $\mathrm{L}$. & Flor morada & \\
\hline Ulmaceae & & $\mathrm{A}$ \\
\hline Celtis loxensis Cc. Berg & Palo blanco & \\
\hline Verbenaceae & & $\mathrm{Ar}$ \\
\hline Citharexylum gentryi Moldenke & Guayacansillo & $\mathrm{Ar}$ \\
\hline Aegiphila sp & Capón & \\
\hline
\end{tabular}

Nota: Forma de vida Árbol (A), Arbusto (Ar), Hierba (H), Epífita (E). 